\title{
A Kohn-Sham system at zero temperature
}

\author{
Horia Cornean \\ Department of Mathematical Sciences \\ Aalborg University \\ Fredrik Bajers Vej 7E \\ DK-9220 Aalborg, Denmark* \\ Kurt Hoke ${ }^{\dagger}$ \\ Hagen Neidhardt ${ }^{\dagger}$ \\ Paul Nicolae Racec ${ }^{\dagger}$ \\ Joachim Rehberg ${ }^{\dagger}$ \\ Weierstrass Institute for \\ Applied Analysis and Stochastics \\ Mohrenstr. 39 \\ D-10117 Berlin, Germany
}

January 17, 2008

\begin{abstract}
An one-dimensional Kohn-Sham system for spin particles is considered which effectively describes semiconductor nanostructures and which is investigated at zero temperature. We prove the existence of solutions and derive a priori estimates. For this purpose we find estimates for eigenvalues of the Schrödinger operator with effective Kohn-Sham potential and obtain $W^{1,2}$-bounds of the associated particle density operator. Afterwards, compactness and continuity results allow to apply Schauder's fixed point theorem. In case of vanishing exchangecorrelation potential uniqueness is shown by monotonicity arguments. Finally, we investigate the behavior of the system if the temperature approaches zero.
\end{abstract}

Subject classification: 34L40, 34L30, 47H05, 81V70

Keywords: Kohn-Sham systems, Schrödinger-Poisson systems, non-linear operators, density operator, zero temperature, Fermi-Dirac distribution

\footnotetext{
*cornean@math.aau.dk

†hoke@wias-berlin.de, neidhard@wias-berlin.de, racec@wias-berlin.de, rehberg@wias-berlin.de
} 


\section{Introduction}

Hohenberg and Kohn have shown in 7 that the ground state of an $N$-body quantum system at zero temperature is completely determined by the particle density. Nowadays that paper is considered as the starting point of the socalled density functional theory. The main advantage of this approach is that the description of an $N$-body quantum problem can be reduced to an effective one-body system. A shortcoming of [7] is that only the existence of such a functional, depending on the particle density, was shown, but the functional was not given explicitely. In [14] Kohn and Sham have indicated such functionals which $N$-electrons look like

$$
\begin{gathered}
\mathcal{E}[u]=-\frac{\hbar^{2}}{2 m} \sum_{n=1}^{N} \int\left|\nabla \varphi_{n}(\mathbf{r})\right|^{2} d^{3} \mathbf{r} \\
-\frac{q^{2}}{4 \pi \epsilon_{0}} \sum_{k=1}^{M} \int \frac{Z_{k} u(\mathbf{r})}{\left|\mathbf{r}-\mathbf{R}_{k}\right|} d^{3} \mathbf{r}+\frac{q^{2}}{2} \iint \frac{u(\mathbf{r}) u\left(\mathbf{r}^{\prime}\right)}{4 \pi \epsilon_{0}\left|\mathbf{r}-\mathbf{r}^{\prime}\right|} d^{3} \mathbf{r} d^{3} \mathbf{r}^{\prime}+\int \varepsilon_{x c}[u](\mathbf{r}) d^{3} \mathbf{r},
\end{gathered}
$$

where $M$ is the number of positive ions, $Z_{k}$ their atomic number, $\mathbf{R}_{k}$ the positions of ions, $q$ is the magnitude of the elementary charge, $\epsilon_{0}$ is the vacuum permittivity and $\varepsilon_{x c}[u]$ is the so-called exchange correlation energy density. The particle density $u$ is given by the expression

$$
u(\mathbf{r}):=2 \sum_{n=1}^{N}\left|\psi_{n}(\mathbf{r})\right|^{2},
$$

where 2 counts for the spin degeneracy of the particles and $\psi_{n}$ are eigenfunctions satisfying the Kohn-Sham equation

$$
\left(-\frac{\hbar^{2}}{2 m} \Delta-\frac{q^{2}}{4 \pi \epsilon_{0}} \sum_{k=1}^{M} \frac{Z_{k}}{\left|\mathbf{r}-\mathbf{R}_{k}\right|}+q^{2} \int \frac{u\left(\mathbf{r}^{\prime}\right)}{4 \pi \epsilon_{0}\left|\mathbf{r}-\mathbf{r}^{\prime}\right|} d^{3} \mathbf{r}^{\prime}+V_{x c}[u](\mathbf{r})\right) \psi_{n}=E_{n} \psi_{n} .
$$

By $V_{x c}[u]$ the so-called exchange correlation potential is denoted, which is given by

The potential

$$
V_{x c}[u](\mathbf{r}):=\frac{\partial\left(\varepsilon_{x c}[u](\mathbf{r})\right)}{\partial u} .
$$

$$
V_{0}(\mathbf{r}):=-\frac{q^{2}}{4 \pi \epsilon_{0}} \sum_{k=1}^{M} \frac{Z_{k}}{\left|\mathbf{r}-\mathbf{R}_{k}\right|}
$$

which is determined by the positive ions, can be regarded as a given external potential. The potential

$$
\varphi(\mathbf{r}):=-q \int \frac{u\left(\mathbf{r}^{\prime}\right)}{4 \pi \epsilon_{0}\left|\mathbf{r}-\mathbf{r}^{\prime}\right|} d^{3} \mathbf{r}^{\prime}
$$


is nothing else as the solution of the Poisson equation

$$
\Delta \varphi(\mathbf{r})=\frac{q u(\mathbf{r})}{\epsilon_{0}} .
$$

So we end up with a Schrödinger operator of the form

$$
H_{V}=-\frac{\hbar^{2}}{2 m} \Delta+V
$$

with the effective Kohn-Sham potential

$$
V:=V_{0}+V_{x c}[u]-q \varphi
$$

where $\varphi$ obeys the Poisson equation (2). The model is very flexible and widely applicable because the exchange correlation term can be well adapted to a great variety of problems. This is one of the reasons why the approach was very successful in the last forty years, and Kohn was awarded the Nobel prize in 1998 for that idea.

We note that the Kohn-Sham system is quite similar to the so-called Hartree-Fock approximation in $N$-body quantum systems [15, 16]. The main difference is that the exchange correlation term for the Hartree-Fock system is not local. However, performing the low density limit one obtains the HartreeFock-Slater approximation [23, 4, 2]. In this case the exchange correlation potential is of the form $V_{x c}[u](x)=-C_{\alpha}|u(x)|^{\alpha}, \alpha=1 / 3$ in three dimensions (3D). For $\alpha=2 / 3$ one gets another interesting approximation which is called the Thomas-Fermi correction. Usually, models of that type are summarized as Schrödinger-Poisson- $X^{\alpha}$ systems, see [1], which fit, of course, into the class of Kohn-Sham systems. In the following we do not restrict ourself to Schrödinger-Poisson- $X^{\alpha}$ systems, but consider a larger class of local and non-local exchange correlation terms including the $X^{\alpha}$-models. Generally, we assume that the exchange correlation is a non-linear mapping acting from the set of densities into the set of potentials obeying a certain continuity condition.

Note that all these considerations are made at zero temperature. An extension of the Hohenberg-Kohn approach to temperatures above zero was proposed by Mermin in [17]. He showed that the expression for the particle density then modifies to

$$
u(\mathbf{r}):=2 \sum_{n=1}^{\infty} \frac{1}{1+e^{\beta\left(E_{n}-\mu\right)}}\left|\psi_{n}(\mathbf{r})\right|^{2},
$$

such that

$$
N=\int u(\mathbf{r}) d^{3} \mathbf{r}=2 \sum_{n=1}^{\infty} \frac{1}{1+e^{\beta\left(E_{n}-\mu\right)}},
$$


where $\beta:=1 / k T$ and $\mu$ is the so called chemical potential.

There are many papers on the numerics of the Kohn-Sham system, but very few on its mathematical analysis. In case of nonzero temperature and bounded domains the system was analyzed in [9] and [10], where existence and a priori estimates were shown. In [20] the Schrödinger-Poisson-Slater system was investigated for a periodic external potential $V_{0}$. The time dynamics of the Schrödinger-Poisson-Slater system was considered in [22].

In the following we are going to investigate the zero and non-zero temperature Kohn-Sham system with a general exchange correlation potential for a planar semiconductor nanostructure. The system reduces essentially to an effective one-dimensional system. Since for one-dimensional systems the eigenvalues are simple, one avoids in this way the occupation problem for the last eigenvalue at zero temperature, if it is degenerated. We show the existence of solutions for such systems at non-zero and zero temperature. In particular, we prove that the solution is unique, if the exchange correlation potential is absent. In the zero temperature case this proof is based on an extension of the monotonicity for the negative particle density operator to non-smooth distribution functions like Fermi-Dirac distribution function at zero temperature, see [8]. Finally, we prove that the non-zero temperature solutions of the Kohn-Sham system converge to those for zero temperature as the temperature goes to zero.

The outline of the paper is as follows: In Section 2 we derive an expression of the effective one-dimensional particle density for a planar semiconductor nanostructure. In Section 3 we introduce the mathematical setup of an one-dimensional Schrödinger-Poisson system and make it mathematically rigorous. Section 4 is devoted to the existence of solutions. In Section 5 we prove the uniqueness of solutions, if the correction term is absent. Finally, in Section 6 we show the convergence of non-zero temperature solutions to zero-temperature ones as the temperature goes to zero.

Notation: In this paper the system is considered on the domain $\Omega:=$ ]0,1[. For this reason we omit for all functional spaces the explicit indication of this interval; e.g. write $L^{1}$ instead of $L^{1}(] 0,1[)$ and so on. We set $W_{0}^{1,2}:=\left\{f \in W^{1,2}: f(0)=f(1)=0\right\}$. The space of antilinear forms on $W_{0}^{1,2}$ is denoted by $W^{-1,2}$. For Banach spaces $X$ and $Z$, we denote by $\mathcal{B}(X ; Z)$ the space of all linear, continuous operators from $X$ into $Z$. If $X=Z$ we write $\mathcal{B}(X)$. Because of the numerous use of $X=L^{2}$ we introduce the abbreviation $\|\cdot\|=\|\cdot\|_{\mathcal{B}\left(L^{2}\right)}$. 


\section{Particle density for planar nanostructures}

We consider a planar semiconductor nanostructure; that is, there is a sequence of layers of different materials along the $x$-direction (i.e. a sequence of quantum wells and barriers) embedded between two thick layers of isolator. Then the wave functions of a particle (electron or hole) are given by

$$
\Psi_{\mathbf{k}_{\perp}, l}(\mathbf{r})=\frac{e^{i \mathbf{k}_{\perp} \mathbf{r}_{\perp}}}{2 \pi} \psi_{l}(x),
$$

and the total energy of the particle is

$$
E=\frac{\hbar^{2} k_{\perp}^{2}}{2 m_{\perp}}+\lambda_{l}
$$

where $\mathbf{r}_{\perp}=(y, z)$ represents the transversal coordinates, $\mathbf{k}_{\perp}=\left(k_{y}, k_{z}\right)$ the transversal wave number and $m_{\perp}$ the effective mass in the transversal direction. The wave functions along the $x$-direction and their corresponding energies correspond to the one-dimensional Schrödinger operator in the effective mass-approximation (Ben-Daniel-Duke form)

$$
H_{V}:=-\frac{\hbar^{2}}{2} \frac{d}{d x}\left(m^{-1} \frac{d}{d x}\right)+V,
$$

where $m=m(x)$ is the position dependent effective mass and $V$ is an effective Kohn-Sham potential to be specified later on. The embedding isolator layers impose homogeneous Dirichlet boundary conditions for the wave function along the $x$-direction, providing a discrete spectrum of energies, $\lambda_{l}$, and defining in such a way a closed system in the $x$-direction.

Quantum mechanically, the particle density is given by a sum over all states of their localization probability multiplied by their occupation probability. For fermions (electrons and holes) the occupation probability is given by the Fermi-Dirac function.

At zero temperature, all states up to the Fermi energy $E_{F}$ are equally occupied, with probability 1 and above $E_{F}$ all states are empty, i.e. occupation probability 0 . Thus, for zero temperature, the particle density is calculated quantum mechanically as

$$
u(\mathbf{r})=2 \underbrace{\int d \mathbf{k}_{\perp} \sum_{l}}_{E \leq E_{F}}\left|\Psi_{\mathbf{k}_{\perp}, l}(\mathbf{r})\right|^{2},
$$

where 2 counts for the spin degeneracy of the particles. Using the expression (77) of the wave functions for planar structures, one has only an $x$ - dependent 
particle density

$$
u(x)=2 \sum_{l=1}^{N_{F}} \frac{\left|\psi_{l}(x)\right|^{2}}{(2 \pi)^{2}} \int_{0}^{k_{\perp, F}^{(l)}} 1 d \mathbf{k}_{\perp},
$$

where the sum runs up to the last occupied level, i.e. $\lambda_{N_{F}} \leq E_{F}$ and the integral is taken up to a maximum value of the transversal wave number, $k_{\perp, F}^{(l)}=\sqrt{\frac{2 m_{\perp}}{\hbar^{2}}\left(E_{F}-\lambda_{l}\right)}-$ depending on $l$. The integral over $d \mathbf{k}_{\perp}$ can be performed, and one obtains the particle density at zero temperature for an effective one-dimensional system [21] as

$$
u(x)=2 \frac{m_{\perp}}{2 \pi \hbar^{2}} \sum_{l=1}^{N_{F}}\left|\psi_{l}(x)\right|^{2}\left(E_{F}-\lambda_{l}\right) .
$$

At temperature $T$ different from zero the particle density is given by

$$
\begin{aligned}
u(\mathbf{r}) & =2 \underbrace{\int d \mathbf{k}_{\perp} \sum_{l}}_{0 \leq E \leq \infty}\left|\Psi_{\mathbf{k}_{\perp}, l}(\mathbf{r})\right|^{2} f_{F D}(E, \mu) \\
& =2 \int_{0}^{\infty} d \mathbf{k}_{\perp} \sum_{l=1}^{\infty}\left|\Psi_{\mathbf{k}_{\perp}, l}(\mathbf{r})\right|^{2} f_{F D}(E, \mu),
\end{aligned}
$$

where $f_{F D}(E, \mu)$ is the Fermi-Dirac distribution function

$$
f_{F D}(E, \mu)=\frac{1}{1+e^{\frac{E-\mu}{k T}}},
$$

$k$ is the Boltzmann constant and $\mu$ is the chemical potential. Inserting (7) and using (86) one obtains an $x$-dependent particle density

$$
u(x)=2 \sum_{l=1}^{\infty} \frac{\left|\psi_{l}(x)\right|^{2}}{(2 \pi)^{2}} \int_{0}^{\infty} f_{F D}\left(\frac{\hbar^{2} k_{\perp}^{2}}{2 m_{\perp}}+\lambda_{l}, \mu\right) d \mathbf{k}_{\perp} .
$$

This corresponds to the general form (16) used later on. Also in this case one can perform the integral over $d \mathbf{k}_{\perp}$, obtaining [3, 25]

$$
u(x)=2 \frac{m_{\perp}}{2 \pi \hbar^{2}} k T \sum_{l=1}^{\infty}\left|\psi_{l}(x)\right|^{2} \ln \left(1+e^{\frac{\mu-\lambda_{l}}{k T}}\right),
$$

which provides the expression for $f_{\beta}(x)$ used in Lemma 6.5 below. Carrying out the limit $T \rightarrow 0$ in (11) one obtains the same expression for the particle density as in the zero temperature limit (10), because

$$
\lim _{T \rightarrow 0} k T \ln \left(1+e^{\frac{\mu-\lambda_{l}}{k T}}\right)=\left(\mu-\lambda_{l}\right) \Theta\left(\mu-\lambda_{l}\right)
$$


( $\Theta$ being the Heaviside function) and it is known that the chemical potential for zero temperature equals the Fermi energy, $\lim _{T \rightarrow 0} \mu=E_{F}$.

\section{Kohn-Sham systems}

\subsection{Kohn-Sham systems in one dimension}

The Kohn-Sham system is a system of equations governing the electrostatic potential $\varphi$ and the particle density $u$ under consideration. Let us consider a system of electrons. The electrostatic potential and the particle density have to obey Poisson's equation

$$
-\frac{d}{d x}\left(\varepsilon \frac{d}{d x} \varphi\right)=D-q u
$$

in the device domain $\Omega=(0,1)$ where $q$ is the magnitude of the elementary charge, and $\varepsilon=\varepsilon(x)$ denotes the dielectric permittivity. The right-hand side of (12) is a charge distribution $D$ of ionized dopants and the particle density $u$ which is defined below, see (16). One has to supplement the Poisson equation (12) by boundary conditions. Usually one chooses inhomogeneous Dirichlet boundary conditions

$$
\varphi(0)=\varphi_{0} \in \mathbb{R} \quad \text { and } \quad \varphi(1)=\varphi_{1} \in \mathbb{R}
$$

which model Ohmic contacts. A straightforward calculation shows that inhomogeneous boundary conditions can be transformed into homogenous boundary conditions. Indeed, introducing the function $\widetilde{\varphi}:[0,1] \longmapsto \mathbb{R}$,

$$
\widetilde{\varphi}:=\varphi_{0}+\frac{\varphi_{1}-\varphi_{0}}{\int_{0}^{1} \frac{1}{\varepsilon(t)} d t} \int_{0}^{x} \frac{1}{\varepsilon(t)} d t
$$

and setting $\phi:=\varphi(x)-\widetilde{\varphi}, x \in[0,1]$, one gets that $\phi$ satisfies the Poisson equation

$$
-\frac{d}{d x} \varepsilon \frac{d}{d x} \phi=D-q u
$$

and obeys the homogeneous Dirichlet boundary conditions

$$
\phi(0)=0 \quad \text { and } \quad \phi(1)=0 .
$$

This gives rise to the Poisson operator $\mathcal{P}:=-\frac{d}{d x} \varepsilon \frac{d}{d x}$, supplemented by homogeneous Dirichlet boundary conditions. 
The particle density $u$ is computed by the quantum mechanical expression

$$
u(V)(x)=2 \sum_{l=1}^{\infty} f\left(\lambda_{l}(V)-\mu_{f}(V)\right)\left|\psi_{l}(V)(x)\right|^{2}, \quad x \in(0,1),
$$

where 2 counts for the spin degeneracy, $f$ is a distribution function (to be specified later on), $\lambda_{l}=\lambda_{l}(V)$ are the eigenvalues and $\psi_{l}=\psi_{l}(V)$ are the corresponding $L^{2}$-normalized orthogonal eigenfunctions of the Schrödinger operator $H_{V}$, cf. (9)). The chemical potential $\mu_{f}(V)$ is determined by the condition

$$
2 \sum_{l=1}^{\infty} f\left(\lambda_{l}(V)-\mu_{f}(V)\right)=N
$$

where $N$ is the number of particles (electrons)- which is considered within this paper as given and fixed, see ([6). The effective Kohn-Sham potential $V$ depends on the particle densities and splits up in the following way

$$
V=V(u)=\Delta E+V_{x c}(u)-q \varphi,
$$

where $\varphi$ denotes the electrostatic potential. The given external potential $\Delta E$ represents the band-edge offsets of the nanostructure materials. $V_{x c}$ is the exchange-correlation potential, which depends on the particle density, see Section 1 .

It is a widely discussed question how to supplement the Schrödinger operators (9) by suitable boundary conditions (see [5, 12, 13]). We choose homogeneous Dirichlet boundary conditions

$$
\psi(0)=0 \quad \text { and } \quad \psi(1)=0
$$

for all $\psi$ in the domain of the Schrödinger operator $H_{V}$. They are assisted by the fact that if we assume a device structure which confines the particles (i.e. closed system), then the particle densities vanish on the boundary of $\Omega$.

\subsection{Rigorous mathematical formulation of the problem}

In this section we give a mathematical formulation of the Kohn-Sham system; in particular, we make precise in which spaces the corresponding operators act and the solutions are chosen. In view of typical applications [6], our mathematical model must necessarily cover semiconductor heterostructures, i.e., the coefficients of the Schrödinger and the Poisson operator are in general discontinuous. This forecloses that the domain of the Schrödinger operator is not lying in $W^{2,2}$ what is natural elsewhere, see e.g. [18, 19]. Fortunately, in the one-dimensional case the $W^{1,2}$-calculus already leads to satisfactory results. Let us introduce the Poisson operator. 
Assumption 3.1. The dielectric permittivity $\varepsilon$ is a real, non-negative function obeying $\varepsilon \in L^{\infty}$ and $\frac{1}{\varepsilon} \in L^{\infty}$.

Definition 3.2. Let Assumption 3.1 be satisfied. We define the Poisson operator $\mathcal{P}: W_{0}^{1,2} \longmapsto W^{-1,2}$ by

$$
<\mathcal{P} v, w>=\int_{0}^{1} \varepsilon(x) v^{\prime}(x) \overline{w^{\prime}(x)} d x, \quad u, w \in W_{0}^{1,2},
$$

where here and in the sequel $<\cdot, \cdot>$ denotes the dual pairing between $W_{0}^{1,2}$ and $W^{-1,2}$.

One easily estimates

$$
|<\mathcal{P} v, w>| \leq\|\varepsilon\|_{L^{\infty}}\|v\|_{W^{1,2}}\|w\|_{W^{1,2}}
$$

for $u, w \in W_{0}^{1,2}$. Consequently, $\mathcal{P}: W_{0}^{1,2} \mapsto W^{-1,2}$ is well defined and continuous. Furthermore, we have

$$
\|\phi\|_{W_{0}^{1,2}}^{2} \leq\|2 / \varepsilon\|_{L^{\infty}} \int_{0}^{1} \varepsilon(x)\left|\phi^{\prime}(x)\right|^{2} d x, \quad \phi \in W_{0}^{1,2},
$$

what implies

$$
\|\phi\|_{W_{0}^{1,2}}^{2} \leq\|2 / \varepsilon\|_{L^{\infty}}<\mathcal{P} \phi, \phi>
$$

for $\phi \in W_{0}^{1,2}$. Hence, by the Lax-Milgram lemma, the inverse operator $\mathcal{P}^{-1}: W^{-1,2} \longmapsto W_{0}^{1,2}$ exists and its norm does not exceed $\|2 / \varepsilon\|_{L^{\infty}}$.

Assumption 3.3. The density of ionized dopants $D$ is a 'real distribution' from $W^{-1,2}$, what means that it takes real values if applied to real elements from $W_{0}^{1,2}$.

Definition 3.4. Let Assumptions 3.1 and 3.3 be satisfied. Further, suppose $u \in L^{1} \hookrightarrow W^{-1,2}$. We say that $\varphi \in W^{1,2}$ is a solution of the Poisson equation (12) obeying the inhomogeneous Dirichlet boundary conditions (13) if $\phi:=\varphi-\widetilde{\varphi} \in W_{0}^{1,2}$ satisfies

$$
\mathcal{P} \phi=D-q u
$$

We set

$$
\varphi(u):=\widetilde{\varphi}+\mathcal{P}^{-1}(D-q u) .
$$

Next we are going to introduce the Schrödinger operator.

Assumption 3.5. The effective mass $m$ is a real, non-negative function obeying $m \in L^{\infty}$ and $\frac{1}{m} \in L^{\infty}$. 
Definition 3.6. Let Assumption 3.5 be satisfied. If $V \in L^{1}$ is real valued, then the Schrödinger operator $H_{V}: W_{0}^{1,2} \longmapsto W^{-1,2}$ corresponding to the potential $V$ is defined by

$$
<H_{V} v, w>=\frac{\hbar^{2}}{2} \int_{0}^{1} \frac{1}{m(x)} v^{\prime}(x) \bar{w}^{\prime}(x) d x+\int_{0}^{1} V(x) v(x) \bar{w}(x) d x,
$$

$v, w \in W_{0}^{1,2}$. The definition is justified, because $W_{0}^{1,2}$ continuously embeds into $L^{\infty}(0,1)$. Thus, the second term on the right hand side is always finite and defines a continuous sesquilinear form on $W_{0}^{1,2}$. The operator with zero potential will be denoted by $H_{0}$ in the sequel. The restriction of the operators just introduced to other range spaces, in particular, to $L^{2}(0,1)$, we also denote by $H_{V}$.

For any real valued $V \in L^{1}(0,1)$, the restriction of $H_{V}$ to the range space $L^{2}(0,1)$ is selfadjoint and has a complete orthonormal system of eigenfunctions. All eigenvalues are then real and simple.

Definition 3.7. We say that a continuous function $f: \mathbb{R} \mapsto[0, \infty[$ is from the class $\mathcal{D}$, if one of the following conditions is satisfied:

(i) there is a $t \in]-\infty, \infty$ [ such that $f$ is strictly monotonously decreasing on the interval $]-\infty, t[$ and zero on $[t, \infty[$,

(ii) the function $f$ obeys $f(s)>0$ for any $s \in \mathbb{R}$ and

$$
\sup _{s \in[1, \infty[} f(s) s^{2}<\infty
$$

is valid.

Assumption 3.8. In the sequel we assume that all occurring distribution functions are from the space $\mathcal{D}$.

Remark 3.9. Let us explicitely note that in contrast to other papers besides continuity no further regularity assumptions are imposed on the distribution functions. Only this allows to include the zero temperature case, see Chapters 6.

Lemma 3.10. Let Assumptions 3.5 and 3.8 be satisfied. If $\left\{\lambda_{l}(V)\right\}_{l}$ are the eigenvalues of the Schrödinger operator $H_{V}$ with real potential $V \in L^{1}$, then for every $N \in[1, \infty[$ there is exactly one real number $E \in \mathbb{R}$ which satisfies

$$
2 \sum_{l} f\left(\lambda_{l}(V)-E\right)=N
$$

This real number is called the chemical potential and is denoted by $\mu_{f}(V)$. 
Proof. For every $f \in \mathcal{D}$ and every $E \in \mathbb{R}$ the expression $\sum_{l} f\left(\lambda_{l}(V)-E\right)$ is finite, $\lim _{E \mapsto-\infty} \sum_{l} f\left(\lambda_{l}(V)-E\right)=0$ and $\lim _{E \mapsto \infty} \sum_{l} f_{l}(\lambda(V)-E)=\infty$. Moreover, it is not hard to see that on the set $\left\{E \in \mathbb{R}: \sum_{l} f\left(\lambda_{l}(V)-E\right) \neq 0\right\}$ the function $E \rightarrow \sum_{l} f\left(\lambda_{l}(V)-E\right)$ is strictly increasing.

Remark 3.11. In the following we assume that the number of particles in the Kohn-Sham system is always fixed by $N \in[1, \infty[$ without indicating this explicitly.

Definition 3.12. Let Assumptions 3.5] and 3.8 be satisfied. Further, let $\left\{\psi_{l}(V)\right\}_{l}$ and $\left\{\lambda_{l}(V)\right\}_{l}$ be the eigenfunctions and eigenvalues of the Schrödinger operator $H_{V}$ with real potential $V \in L^{1}$ and let $\mu_{f}(V)$ be the chemical potential. Then

$$
\mathcal{N}_{f}(V)(x):=2 \sum_{l} f\left(\lambda_{l}(V)-\mu_{f}(V)\right)\left|\psi_{l}(V)(x)\right|^{2}, \quad x \in[0,1]
$$

defines an operator $\mathcal{N}_{f}: L^{1} \longmapsto L^{1}$ which is called the particle density operator.

Remark 3.13. $\mathcal{N}_{f}(V)$ obviously satisfies

$$
\int_{0}^{1} \mathcal{N}_{f}(V) d x=2 \sum_{l=1}^{\infty} f\left(\lambda_{l}(V)-\mu_{f}(V)\right)=N .
$$

Assumption 3.14. a) The potential $\Delta E$ is a real-valued $L^{1}$ function.

b) The exchange-correlation term in its dependence on the particle densities, i.e. the mapping $u \longmapsto V_{x c}(u)$, is a continuous and bounded mapping from $L^{1}$ into $L^{1}$. This assumption covers the Hartree-Fock-Slater and Thomas-Fermi exchange-correlation terms.

Definition 3.15. Let Assumptions [3.1, 3.3, 3.5, 3.8 and 3.14 be satisfied. The pair $\{\varphi, u\} \in W^{1,2} \times L^{1}$ is called a solution of the Kohn-Sham system with distribution function $f$, if $\varphi$ solves the Poisson equation (12) with inhomogeneous Dirichlet boundary conditions (13) and the particle density $u$ is given by

$$
u:=\mathcal{N}_{f}\left(\Delta E+V_{x c}(u)-q \varphi\right) .
$$

\section{Existence of solutions}

In this section we are going to show that the Kohn-Sham system always admits a solution. As in [9, 10] Schauder's fixed point theorem is used, what 
requires several estimates (e.g. eigenvalues of the Schrödinger operator, $W^{1,2}$ bounds of the particle density operator). To assure its applicability, we first establish some prerequisites.

Definition 4.1. For $m \in L^{\infty}$ we set $\underline{m}:=\max \left(1, \frac{2\|m\|_{L^{\infty}}}{\hbar^{2}}\right)$.

Remark 4.2. Recognizing that $\underline{m}$ has been defined such that $1 / \underline{m}$ is a monotonicity constant of the operator $H_{1}=H_{0}+1: W_{0}^{1,2} \mapsto W^{-1,2}$, the LaxMilgram lemma shows, that the norm of the inverse operator is not larger than $\underline{m}$,

$$
\|\psi\|_{W_{0}^{1,2}}^{2} \leq \underline{m}<\left(H_{0}+1\right) \psi, \psi>, \quad\left\|\left(H_{0}+1\right)^{-1}\right\|_{\mathcal{B}\left(W^{-1,2}, W_{0}^{1,2}\right)} \leq \underline{m} .
$$

By some calculations one finds

$$
\|\psi\|_{L^{\infty}} \leq \sqrt{2}\|\psi\|_{W_{0}^{1,2}}^{\frac{1}{2}}\|\psi\|_{L^{2}}^{\frac{1}{2}}
$$

which proves the continuous embedding $W_{0}^{1,2} \hookrightarrow L^{\infty}$.

The following proposition allows to compare the eigenvalues of the Schrödinger operators $H_{V}$ and $H_{0}$ and, additionally, provides a comparison between the operators $\left(H_{0}+1\right)^{-1 / 2}$ and $\left(H_{V}-\rho\right)^{-1 / 2}$. Both we will need later on as technical instruments.

Proposition 4.3. Let Assumption 3.5 be satisfied and let $V \in L^{1}$ be realvalued. Then the following holds:

(i) The eigenvalues $\lambda_{l}(V)$ of the operator $H_{V}$ can be estimated as follows:

$$
\frac{1}{2}\left(\varsigma_{l}+1\right)+\rho_{V} \leq \lambda_{l}(V) \leq \frac{3}{2}\left(\varsigma_{l}+1\right)-\rho_{V}-2, \quad l=1,2, \ldots
$$

where the $\varsigma_{l}$ are the eigenvalues of the operator $H_{0}$ and $\rho_{V}$ is given by

$$
\rho_{V}=-2\|V\|_{L^{1}}^{2} \underline{m}-1 .
$$

(ii) For $\rho \leq \rho_{V}$ the spectrum of $\left(H_{V}-\rho\right)^{-1}$ is contained in $[0,2]$ and

$$
\left\|\left(H_{V}-\rho\right)^{-\frac{1}{2}}\left(H_{0}+1\right)^{\frac{1}{2}}\right\|=\left\|\left(H_{0}+1\right)^{\frac{1}{2}}\left(H_{V}-\rho\right)^{-\frac{1}{2}}\right\| \leq \sqrt{2} .
$$

A proof of this is to be found in [9], see Prop. 3.3. 
Remark 4.4. The form which defines $H_{0}$ may be estimated as follows:

$$
\begin{aligned}
& \frac{\hbar^{2}}{2} \underset{x \in(0,1)}{\operatorname{essinf}}\left\{\frac{1}{m}\right\} \int_{0}^{1}\left|v^{\prime}(x)\right|^{2} d x \leq \\
& \quad \frac{\hbar^{2}}{2} \int_{0}^{1} m(x)^{-1}\left|v^{\prime}(x)\right|^{2} d x \leq \frac{\hbar^{2}}{2} \underset{x \in(0,1)}{\operatorname{ess} \sup }\left\{\frac{1}{m}\right\} \int_{0}^{1}\left|v^{\prime}(x)\right|^{2} d x .
\end{aligned}
$$

Thus, the eigenvalues of $H_{0}$ compare by the minimax principle from below and above in an obvious manner with the eigenvalues of $-\frac{d^{2}}{d x^{2}}-$ combined with a homogeneous Dirichlet condition.

The reader should notice that Proposition 4.3 gives uniform bounds with respect to $L^{1}$-bounded sets of potentials.

From Proposition 4.3 we can deduce the following

Lemma 4.5. Let Assumption 3.5 be satisfied and let $\mathcal{M} \subset L^{1}$ be a bounded set of real-valued potentials. If

$$
\rho<\rho_{\mathcal{M}}:=\inf _{V \in \mathcal{M}} \rho_{V}=-2 \underline{m} \sup _{V \in \mathcal{M}}\|V\|_{L^{1}}^{2}-1,
$$

then the mapping

$$
L^{1} \supset \mathcal{M} \ni V \mapsto\left(H_{V}-\rho\right)^{-1} \in \mathcal{B}\left(L^{2}\right)
$$

is Lipschitz continuous in $V$ with a Lipschitz constant depending on $\mathcal{M}$.

Proof. If $\rho \leq \rho_{\mathcal{M}}$, then $\rho$ belongs to the resolvent set of $H_{V}$ for any $V \in \mathcal{M}$ by Proposition 4.3. Moreover, one has

$$
H_{V}-\rho=H_{V}-\rho_{V}+\rho_{V}-\rho \geq \frac{1}{2} I
$$

since $H_{V}-\rho_{V} \geq \frac{1}{2} I$ for $V \in \mathcal{M}$ by Proposition 4.3. Applying the resolvent equation

$$
\left(H_{V}-\rho\right)-\left(H_{U}-\rho\right)=\left(H_{V}-\rho\right)(U-V)\left(H_{U}-\rho\right),
$$

one obtains

$$
\begin{aligned}
& \left\|\left(H_{V}-\rho\right)^{-1}-\left(H_{U}-\rho\right)^{-1}\right\|= \\
& \quad\left\|\left(H_{V}-\rho\right)^{-1}(U-V)\left(H_{U}-\rho\right)^{-1}\right\| .
\end{aligned}
$$


The latter term may be estimated as follows:

$$
\begin{gathered}
\left\|\left(H_{V}-\rho\right)^{-1}(U-V)\left(H_{U}-\rho\right)^{-1}\right\| \leq\|U-V\|_{\mathcal{B}\left(L^{\infty} ; L^{1}\right)} \times \\
\left\|\left(H_{V}-\rho\right)^{-1 / 2}\right\|\left\|\left(H_{V}-\rho\right)^{-1 / 2}\left(H_{0}+1\right)^{1 / 2}\right\| \times \\
\left\|\left(H_{0}+1\right)^{-1 / 2}\right\|_{\mathcal{B}\left(L^{1} ; L^{2}\right)}\left\|\left(H_{0}+1\right)^{-1 / 2}\right\|_{\mathcal{B}\left(L^{2} ; L^{\infty}\right)} \times \\
\left\|\left(H_{0}+1\right)^{1 / 2}\left(H_{U}-\rho\right)^{-1 / 2}\right\|\left\|\left(H_{U}-\rho\right)^{-1 / 2}\right\| .
\end{gathered}
$$

The factors $\left\|\left(H_{V}-\rho\right)^{-1 / 2}\right\|$ and $\left\|\left(H_{U}-\rho\right)^{-1 / 2}\right\|$ are uniformly bounded in $U, V$ due to (22). $\left\|\left(H_{V}-\rho\right)^{-1 / 2}\left(H_{0}+1\right)^{1 / 2}\right\|,\left\|\left(H_{0}+1\right)^{1 / 2}\left(H_{U}-\rho\right)^{-1 / 2}\right\|$ are uniformly bounded by (21). Furthermore, $\left\|\left(H_{0}+1\right)^{-1 / 2}\right\|_{\mathcal{B}\left(L^{2} ; L^{\infty}\right)}$ is finite by the embedding $W_{0}^{1,2} \hookrightarrow L^{\infty}$. This implies $\left\|\left(H_{0}+1\right)^{-1 / 2}\right\|_{\mathcal{B}\left(L^{1} ; L^{2}\right)}<\infty$ by duality. Finally, $\|U-V\|_{\mathcal{B}\left(L^{\infty} ; L^{1}\right)}$ is identical with $\|U-V\|_{L^{1}}$.

Corollary 4.6. Let Assumption 3.5 be satisfied. If $\left\{V_{n}\right\}_{n}$ converges to $V$ in $L^{1}$, then the operator sequence $\left\{H+V_{n}\right\}_{n \in \mathbb{N}}$ converges in the norm resolvent sense to $H+V$. In particular, the eigenvalues and the eigenprojections of $H+V_{n}$ converge to the corresponding eigenvalues and eigenprojections of $H+V$.

The proof follows from the preceding corollary and a well known perturbation theorem, see [11, Ch. IV.3.4].

Lemma 4.7. Let Assumptions 3.5 and 3.8 be satisfied.

(i) For any bounded set of real potentials in $L^{1}$ the set of chemical potentials is also bounded. Additionally, this bound can be taken even uniform with respect to any subset $\mathcal{D}_{1} \subset \mathcal{D}$ of distribution functions $f$ obeying in addition

$$
\sup _{f \in \mathcal{D}_{1}} \sup _{t \in[1, \infty[} f(t) t<\infty \quad \text { and } \quad \inf _{f \in \mathcal{D}_{1}} f(a)>0 \quad \text { for one } a \in \mathbb{R} .
$$

(ii) Let $\mathcal{C}:=\left\{f_{j}\right\}_{j=1}^{\infty}$ be a sequence of functions from $\mathcal{D}_{1}$ which converges uniformly on bounded intervals to a function $f \in \mathcal{D}$ as $j \rightarrow \infty$. If $V_{j} \mapsto V$ in $L^{1}$, then $\lim _{j \rightarrow \infty} \mu_{f_{j}}\left(V_{j}\right)=\mu_{f}(V)$.

Proof. (i) By the monotonicity of $f$, one has $\sum_{l} f\left(\lambda_{l}(V)-E\right) \geq k \inf _{f \in \mathcal{C}} f(a)$ if $k$ items $\lambda_{l}-E$ are situated below $a$. This means $\sum_{l} f\left(\lambda_{l}(V)-E\right) \mapsto \infty$ for $E \mapsto \infty$ uniform within $\mathcal{C}$. Thus, the chemical potential $\mu_{f}(V)$ have to be bounded from above by the monotonicity of $f$ and (20), uniformly within the class $\mathcal{C}$. On the other hand, if $E \leq \inf _{l} \inf \operatorname{spec}\left(H_{V_{l}}\right)-1$, then one can estimate

$$
\sum_{l} f\left(\lambda_{l}(V)-E\right) \leq \sup _{f \in \mathcal{C}} \sup _{t \in[1, \infty[} f(t) t \sum_{l}\left(\lambda_{l}(V)-E\right)^{-1}
$$


what -again by (201) - tends to zero for $E \mapsto-\infty$ uniformly for $f \in \mathcal{C}$ and uniform over sets of potentials which are bounded in $L^{1}$.

(ii) First, the chemical potentials are uniformly bounded, due to (i). Thus, the eigenvalues of the operators $H_{V_{j}-\mu_{f_{j}}\left(V_{j}\right)}$ admit uniform bounds as in Proposition 4.3. Assume that the assertion was not true; then for a subsequence $\left\{V_{k}\right\}_{k}$ one has

$$
\left|\mu_{f_{k}}\left(V_{k}\right)-\mathcal{E}_{f}(V)\right|>\epsilon>0 .
$$

Because the chemical potentials form a bounded set, we may again pass to a subsequence $\left\{V_{n}\right\}_{n}$, and suppose

$$
\lim _{n \rightarrow \infty} \mu_{f_{n}}\left(V_{n}\right)=E \neq \mu_{f}(V) .
$$

We will now show that this leads to

$$
\lim _{n \mapsto \infty} \sum_{l} f_{n}\left(\lambda_{l}\left(V_{n}\right)-\mu_{f_{n}}\left(V_{n}\right)\right)=\sum_{l} f\left(\lambda_{l}(V)-E\right) .
$$

First, it follows from (23) and Proposition 4.3 that for any $\delta>0$ there is a number $l_{0}$ such that

$$
\sum_{l>l_{0}} f_{n}\left(\lambda_{l}\left(V_{n}\right)-\mu_{f_{n}}\left(V_{n}\right)\right)<\delta \text { and } \sum_{l>l_{0}} f\left(\lambda_{l}(V)-E\right)<\delta
$$

uniformly for all $n$. The remaining eigenvalues $\lambda_{l}\left(V_{n}\right)$ for $l \leq l_{0}$ and all $n$ lie in a bounded interval and, additionally, due to Corollary 4.6, one has $\lim _{n \mapsto \infty} \lambda_{l}\left(V_{n}\right)=\lambda_{l}(V)$ for every $l$. Thus, according to (25), (27) and the uniform convergence of $\left\{f_{j}\right\}_{j}$ to $f$ on bounded intervals as $j \rightarrow \infty$, the term

$$
\left|\sum_{l} f_{n}\left(\lambda_{l}\left(V_{n}\right)-\mu_{f_{n}}\left(V_{n}\right)\right)-\sum_{l} f\left(\lambda_{l}(V)-E\right)\right|
$$

becomes smaller than $3 \delta$ for sufficiently large $n$ and arbitrarily chosen $\delta>0$. This implies (26), but (26) must be false: the terms on the left hand side all equal $N$, what cannot be true for the right hand side due to (25) and Lemma 3.10, Hence, (24) is wrong, what proves (ii).

Remark 4.8. The lemma shows in particular that the chemical potentials continuously depend on the potential.

Theorem 4.9. Let Assumptions 3.5 and 3.8 be satisfied and let $\mathcal{M}$ be a bounded set of real potentials in $L^{1}$. Then the following holds: 
(i) The image $\mathcal{N}_{f}(\mathcal{M}), f \in \mathcal{D}$, is a bounded set in $W_{0}^{1,2}$. The bound may be taken uniformly with respect to any set $\mathcal{D}_{2} \subset \mathcal{D}$ of distribution functions $f$ which satisfy the additional conditions

$$
\sup _{f \in \mathcal{D}_{2}} \sup _{t \in[0, \infty[} f(t) t(t+1)<\infty \quad \text { and } \quad \inf _{f \in \mathcal{D}_{2}} f(a)>0 \quad \text { for one } \quad a \in \mathbb{R} .
$$

(ii) The particle density operator $\mathcal{N}_{f}: L^{1} \mapsto L^{1}, f \in \mathcal{D}$, is continuous.

Proof. (i) For $V \in \mathcal{M}$ we get

$$
\begin{aligned}
& \left\|\mathcal{N}_{f}(V)\right\|_{W_{0}^{1,2}}=\left\|2 \sum_{l} f\left(\lambda_{l}(V)-\mu_{f}(V)\right)\left|\psi_{l}\right|^{2}\right\|_{W_{0}^{1,2}} \leq \\
& \quad 2 \sum_{l} f\left(\lambda_{l}(V)-\mu_{f}(V)\right)\left\|\left|\psi_{l}\right|^{2}\right\|_{W_{0}^{1,2}} \leq 4 \sum_{l} f\left(\lambda_{l}(V)-\mu_{f}(V)\right)\left\|\psi_{l}\right\|_{W_{0}^{1,2}}^{2}
\end{aligned}
$$

where in the last step we used $\left\||\psi|^{2}\right\|_{W_{0}^{1,2}} \leq 2\|\psi\|_{W_{0}^{1,2}}^{2}$ for all $\psi \in W_{0}^{1,2}$. We estimate the terms in (29):

$$
\begin{aligned}
& \left\|\psi_{l}\right\|_{W_{0}^{1,2}}^{2} \leq \underline{m}\left\|\left(H_{0}+1\right)^{\frac{1}{2}} \psi_{l}\right\|_{L^{2}}^{2} \leq \\
& \quad \underline{m}\left\|\left(H_{0}+1\right)^{\frac{1}{2}}\left(H_{V}-\rho\right)^{-\frac{1}{2}}\right\|^{2}\left\|\left(H_{V}-\rho\right)^{\frac{1}{2}} \psi_{l}\right\|_{L^{2}}^{2} \leq 2 \underline{m}\left(\lambda_{l}(V)-\rho\right),
\end{aligned}
$$

(see (21)) where $\rho<\rho_{\mathcal{M}}$ where $\rho_{\mathcal{M}}$ given by Lemma 4.5 is a uniform lower bound for the spectra of the operators $H_{V}$ with $V \in \mathcal{M}$. Thus, (29) is not larger than

$$
\begin{aligned}
& \left\|\mathcal{N}_{f}(V)\right\|_{W_{0}^{1,2}} \leq 8 \underline{m} \sum_{l} f\left(\lambda_{l}(V)-\mu_{f}(V)\right)\left(\lambda_{l}(V)-\rho\right) \leq \\
& \quad 8 \underline{m} \sum_{l} f\left(\lambda_{l}(V)-\mu_{f}(V)\right)\left[\left(\mu_{f}(V)-\rho\right)+\left(\lambda_{l}(V)-\mu_{f}(V)\right)\right] \leq \\
& \quad 8 \underline{m} N\left|\mu_{f}(V)-\rho\right|+8 \underline{m} \sum_{l: \lambda_{l}(V)-\mu_{f}(V) \geq 0} f\left(\lambda_{l}(V)-\mu_{f}(V)\right)\left(\lambda_{l}(V)-\mu_{f}(V)\right) .
\end{aligned}
$$

The last sum may be estimated by

$$
\sup _{t \in[0, \infty[} f(t) t(t+1) \sum_{l: \lambda_{l}(V)-\mu_{f}(V) \geq 0}\left(\lambda_{l}(V)-\mu_{f}(V)+1\right)^{-1} .
$$

Obviously, the condition (28) is stronger than (23); thus, the set of chemical potentials is uniformly bounded for $f \in \mathcal{C}$ and $V \in \mathcal{M}$. This, together with the eigenvalue estimates (20) and Remark 4.4 proves (i). 
(ii) According to Corollary 4.6 and Lemma 4.7 the eigenvalues and the corresponding chemical potentials depend continously on $V \ni L^{1}$. Furthermore, if $\left\{V_{j}\right\}_{j}$ converges in $L^{1}$ to $V$, then - due to Corollary 4.6 - the (onedimensional) eigenprojections $P_{j, k}=\left\langle\cdot, f_{j, k}\right\rangle f_{j, k}$ converge to the corresponding eigenprojections $P_{k}=\left\langle\cdot, f_{k}\right\rangle f_{k}$. Applying these eigenprojections to the vector $f_{k}$, one easily obtains

$$
\left|\left\langle f_{k}, f_{j, k}\right\rangle\right| \mapsto 1 \quad \text { and } \quad\left\langle f_{k}, f_{j, k}\right\rangle f_{j, k} \mapsto f_{k} \quad \text { in } L^{2} \quad \text { for } \quad j \mapsto \infty \text {. }
$$

From this it is not hard to see that $\left|f_{j, k}\right|^{2} \mapsto\left|f_{k}\right|^{2}$ in $L^{1}$. Observing that for sufficiently large $M$

$$
\left\|\sum_{l \geq M} f\left(\lambda_{l}(V)-\mu_{f}(V)\right)\left|\psi_{l}(V)\right|^{2}\right\|_{L^{1}} \leq \sum_{l \geq M} f\left(\lambda_{l}(V)-\mu_{f}(V)\right)
$$

can be made arbitrarily small uniformly over an $L^{1}$-bounded set of potentials $V$, one obtains (ii).

Corollary 4.10. Let Assumptions 3.5 and 3.8 be satisfied. If $X$ is a Banach space continuously injecting into $L^{1}$ such that $W_{0}^{1,2}$ compactly embeds into $X$, then the particle density operator $\mathcal{N}_{f}: L^{1} \mapsto X$ is well defined and continuous.

Proof. That it is well defined follows from the embedding $W_{0}^{1,2} \hookrightarrow X$. Assume that the continuity does not hold; then there must be a sequence $\left\{V_{n}\right\}_{n}$ converging in $L^{1}$ to $V$ such that

$$
\left\|\mathcal{N}_{f}\left(V_{n}\right)-\mathcal{N}_{f}(V)\right\|_{X}>\epsilon>0
$$

The statement (i) of the foregoing lemma tells us that $\left\{\mathcal{N}_{f}\left(V_{n}\right)\right\}_{n}$ is bounded in $W^{1,2}$. Thus, by the compactness of the embedding $W^{1,2} \hookrightarrow X$ there must be a subsequence $\left\{V_{k}\right\}_{k}$ such that $\mathcal{N}_{f}\left(V_{k}\right)$ converges in $X$ to an element $\mathcal{W}$. But $\mathcal{N}_{f}\left(V_{k}\right)$ converges to $\mathcal{N}_{f}(V)$ in $L^{1}$. Thus $\mathcal{W}$ must equal $\mathcal{N}_{f}(V)$ by the continuous injection $X \hookrightarrow L^{1}$, what contradicts (30).

To prove existence of solutions of the Kohn-Sham system we will introduce an appropriate subset of $L^{1}$ together with a suitable mapping $\Phi$ from this set into itself. $\Phi$ will be constructed such that the solutions to the Kohn-Sham system coincide with the fixed points of this mapping.

Definition 4.11. We set

$$
L_{N}^{1}:=\left\{u \in L^{1}: u \geq 0, \quad \int_{0}^{1} u d x=N\right\} .
$$


Let Assumptions 3.1, 3.3, 3.5, 3.8 and 3.14 be satisfied. Then we define the mapping $\Phi_{f}: L_{N}^{1} \rightarrow L_{N}^{1}$ by

$$
\Phi_{f}(u):=\mathcal{N}_{f}\left(\Delta E+V_{x c}(u)-q \varphi(u)\right)
$$

where $\varphi(u)$ denotes the $u$-dependent solution to Poisson's equation given by (19).

The task now is to verify that $\Phi_{f}$ has a fixed point. We will use Schauder's theorem to achieve this.

Theorem 4.12. Let Assumptions [3.1, 3.3, 3.5, 3.8 and 3.14 be satisfied. Then

(i) the pair $\{\varphi(u), u\}$ is a solution of the Kohn-Sham system if and only if $u \in L_{N}^{1}$ is a fixed point of $\Phi_{f}$, where $\varphi(u)$ is given by (19) and

(ii) the mapping $\Phi_{f}$ has a fixed point.

Proof. (i) The first part of theorem follows immediately from the definition of the mappings $\mathcal{N}_{f}$ and $\Phi_{f}$.

(ii) To prove the second part we note that $L_{N}^{1}$ is a closed, bounded and convex set, which is mapped by $\Phi_{f}$ into itself (Def. 4.11).

Continuity: Since $L^{1} \hookrightarrow W^{-1,2}$, the solution $\varphi$ to Poisson's equation depends continuously (in $W_{0}^{1,2}$ ) on $u$. Hence, due to Assumption 3.14, the mapping $L^{1} \ni u \mapsto \Delta E+V_{x c}(u)-q \varphi(u) \in L^{1}$ is continuous. Theorem 4.9 then implies the continuity of $\Phi_{f}$.

Compactness: According to Theorem 4.9, the image of a $L^{1}$-bounded set of potentials is bounded in the space $W^{1,2}$. The compactness of the embedding $W^{1,2} \hookrightarrow L^{1}$ yields the compactness of $\Phi_{f}$. $L_{N}^{1}$.

Thus, due to Schauder's fixed point theorem, $\Phi_{f}$ has a fixed point in

\section{$5 \quad$ Particle density operator and monotonicity}

In this section we want to show some additional properties of the particle density operator. For this it is necessary to restrict some considerations to the real parts of spaces which were up to now considered as complex ones. We indicate this by an additional subscript $\mathbb{R}$, e.g. $L_{\mathbb{R}}^{\infty}$. The upcoming results are based on the following theorem. 
Theorem 5.1. Let $H$ be a self-adjoint operator in the separable Hilbert space $\mathcal{H}$ with compact resolvent and let $U$ and $V$ be bounded, self-adjoint operators on $\mathcal{H}$. If $f: \mathbb{R} \longrightarrow \mathbb{R}$ is a Borel measurable function such that $f(H+U)$ and $f(H+V)$ are trace class operators, then the formula

$$
\begin{aligned}
& \operatorname{tr}([f(H+U)-f(H+V)](U-V))= \\
& \sum_{k, l=1}^{\infty}\left(f\left(\lambda_{k}\right)-f\left(\mu_{l}\right)\right)\left(\lambda_{k}-\mu_{l}\right)\left|\left\langle\psi_{k}, \xi_{l}\right\rangle\right|^{2}
\end{aligned}
$$

is valid. Here $\left\{\lambda_{k}\right\}_{k}\left(\left\{\mu_{l}\right\}_{l}\right)$ is the sequence of eigenvalues of $H+U(H+$ $V)$ and $\left\{\psi_{k}\right\}_{k}\left(\left\{\xi_{l}\right\}_{l}\right)$ is an - orthornormalized - sequence of corresponding eigenvectors.

The proof is given in [8].

Corollary 5.2. If Assumptions 3.5 and 3.8 are satisfied, then the mappings $L_{\mathbb{R}}^{1} \ni V \rightarrow \mathcal{N}_{f}(V) \in L_{\mathbb{R}}^{\infty}$ and $W_{0, \mathbb{R}}^{1,2} \ni V \rightarrow \mathcal{N}_{f}(V) \in W_{\mathbb{R}}^{-1,2}$ are monotone.

Proof. We specify the Hilbert space $\mathcal{H}$ to $L^{2}$ and first consider potentials $U, V \in L_{\mathbb{R}}^{\infty}$, which are identified with the induced multiplication operators on $L^{2}$. Replacing $U, V$ in the preceding theorem by $U-\mu_{f}(U), V-\mu_{f}(V)$ and observing

$$
\begin{aligned}
& \operatorname{tr}\left(\left[f\left(H+U-\mu_{f}(U)\right)-f\left(H+V-\mu_{f}(V)\right)\right]\left(\mu_{f}(U)-\mu_{f}(V)\right)\right)= \\
& \operatorname{tr}\left(\left[f\left(H+U-\mu_{f}(U)\right)-f\left(H+V-\mu_{f}(V)\right)\right]\right)\left(\mu_{f}(U)-\mu_{f}(V)\right)=0
\end{aligned}
$$

one obtains

$$
\begin{aligned}
& \int_{0}^{1}\left(\mathcal{N}_{f}(U)-\mathcal{N}_{f}(V)\right)(U-V) d x= \\
& 2 \operatorname{tr}\left(\left[f\left(H+U-\mu_{f}(U)\right)-f\left(H+V-\mu_{f}(V)\right)\right](U-V)\right)= \\
& 2 \operatorname{tr}\left(\left[f\left(H+U-\mu_{f}(U)\right)-f\left(H+V-\mu_{f}(V)\right)\right]\left(U-\mu_{f}(U)-V+\mu_{f}(V)\right)\right) .
\end{aligned}
$$

In view of (32) the right hand side is negative because the distribution function $f$ is monotonously decreasing.

Let now $U, V \in L_{\mathbb{R}}^{1}$ be arbitrary. If $\left\{U_{n}\right\}_{n},\left\{V_{n}\right\}_{n}$ are sequences from $L_{\mathbb{R}}^{\infty}$ which converge to $U, V$ in $L^{1}$, respectively, then, on one hand, $\mathcal{N}_{f}\left(U_{n}\right) \mapsto$ $\mathcal{N}_{f}(U)$ and $\mathcal{N}_{f}\left(V_{n}\right) \mapsto \mathcal{N}_{f}(V)$ in $L_{\mathbb{R}}^{\infty}$, due to Corollary 4.10, Because one already knows that $\int_{0}^{1}\left(\mathcal{N}_{f}\left(U_{n}\right)-\mathcal{N}_{f}\left(V_{n}\right)\right)\left(U_{n}-V_{n}\right) d x \leq 0$, one obtains $\int_{0}^{1}\left(\mathcal{N}_{f}(U)-\mathcal{N}_{f}(V)\right)(U-V) d x \leq 0$.

The $W_{0, \mathbb{R}}^{1,2} \leftrightarrow W_{\mathbb{R}}^{-1,2}$ duality extends the $L_{\mathbb{R}}^{\infty} \leftrightarrow L_{\mathbb{R}}^{1}$ duality; thus, the second assertion follows from the first. 
Theorem 5.3. Let Assumptions 3.1, 3.3, 3.5] 3.8 and 3.14 a) be satisfied. If the correlation term $V_{x c}$ is absent, then the Kohn-Sham system has a unique solution $\{\varphi, u\}$.

Proof. It is not hard to see that in this case the system can be written as one equation for the electrostatic potential in the real space $W_{\mathbb{R}}^{-1,2}$, namely

$$
-\frac{d}{d x} \varepsilon \frac{d}{d x} \phi+q \mathcal{N}_{f}(\Delta E-q \widetilde{\varphi}-q \phi)=D
$$

where $\varphi=\widetilde{\varphi}+\phi$. Since the operator $-\frac{d}{d x} \varepsilon \frac{d}{d x}: W_{0, \mathbb{R}}^{1,2} \mapsto W_{\mathbb{R}}^{-1,2}$ is strongly monotone, the operator $-\frac{d}{d x} \varepsilon \frac{d}{d x}+q \mathcal{N}_{f}(\Delta E-q \widetilde{\varphi}-q \cdot): W_{0, \mathbb{R}}^{1,2} \mapsto W_{\mathbb{R}}^{-1,2}$ is also strongly monotone by the foregoing corollary. Additionally, this latter operator is continuous, and hence (33) has a unique solution by the theory of monotone operators, see [24] Ch.26.2.

Corollary 5.4. Let the assumptions of Theorem 5.3 be satisfied. If $\{\varphi, u\}$ is a solution of the Kohn-Sham system, then the electrostatic potential $\phi=\varphi-\widetilde{\varphi}$ satisfies the following a priori estimate:

$$
\|\phi\|_{W_{0}^{1,2}} \leq \frac{1}{M}\left(\|D\|_{W_{0}^{-1,2}}+\gamma_{L^{1} ; W^{-1,2}} N q\right),
$$

where $M$ is the monotonicity constant for the operator $-\frac{d}{d x} \varepsilon \frac{d}{d x}: W_{0, \mathbb{R}}^{1,2} \mapsto$ $W_{\mathbb{R}}^{-1,2}$ and $\gamma_{L^{1} ; W^{-1,2}}$ is the norm of the embedding operator $L^{1} \hookrightarrow W^{-1,2}$.

Proof. Clearly, $\phi$ satisfies the equation

$$
-\frac{d}{d x} \varepsilon \frac{d}{d x} \phi+q \mathcal{N}_{f}\left(\Delta E+V_{x c}(u)-q \widetilde{\varphi}-q \phi\right)=D,
$$

which can be regarded as an equation in $W_{\mathbb{R}}^{-1,2}$, due to Assumption 3.3 and the fact that the dielectric permittivity matrix $\varepsilon$ has real entries, cf. Assumption 3.1. Considering $u$ as fixed, the left hand side is a strongly monotone, continuous operator when acting on $\phi$. We denote it for brevity by $\mathcal{A}$. Its monotonicity constant is at least $M$. Using the strong monotonicity, we may estimate:

$$
\|\mathcal{A} \phi-\mathcal{A} 0\|_{W_{\mathbb{R}}^{-1,2}}\|\phi\|_{W_{0, \mathbb{R}}^{1,2}} \geq|<\mathcal{A} \phi-\mathcal{A} 0, \phi>| \geq M\|\phi\|_{W_{0, \mathbb{R}}^{1,2}}^{2}
$$

what leads to

$$
\begin{gathered}
\|\phi\|_{W_{0, \mathbb{R}}^{1,2}} \leq \frac{1}{M}\left(\|D\|_{W_{\mathbb{R}}^{-1,2}}+q\left\|\mathcal{N}_{f}\left(\Delta E+V_{x c}(u)-q \widetilde{\varphi}\right)\right\|_{W_{\mathbb{R}}^{-1,2}}\right) \leq \\
\leq \frac{1}{M}\left(\|D\|_{W_{\mathbb{R}}^{-1,2}}+q \gamma_{L^{1} ; W_{\mathbb{R}}^{-1,2}}\left\|\mathcal{N}_{f}\left(\Delta E+V_{x c}(u)-q \widetilde{\varphi}\right)\right\|_{L_{\mathbb{R}}^{1}}\right) .
\end{gathered}
$$

Obviously, $\left\|\mathcal{N}_{f}\left(\Delta E+V_{x c}(u)-q \widetilde{\varphi}\right)\right\|_{L_{\mathbb{R}}^{1}}$ equals $N$, what gives the assertion.

Remark 5.5. Note that this estimate depends in no way on the distribution function $f$ (within the class of monotonously decreasing functions, of course). 


\section{Convergence to zero temperature}

In the following we introduce the function $\theta$ by defining

$$
\theta(x)=\left\{\begin{array}{lll}
1 & : & x \leq 1 \\
x & : & x>1
\end{array}\right.
$$

We start with the following technical lemma.

Lemma 6.1. Let Assumptions 3.5 and 3.8 be satisfied. Further, let $\mathcal{C}:=$ $\left\{f_{j}\right\}_{j=1}^{\infty} \subset \mathcal{D}_{2}$, cf. Theorem 4.9, and $f \in \mathcal{D}$ such that

$$
\lim _{j \mapsto \infty} \sup _{x \in[a, \infty[}\left|f_{j}(x)-f(x)\right| \theta(x)=0
$$

holds for every $a \in]-\infty,-1\left[\right.$. If $\left\{V_{j}\right\}_{j=1}^{\infty}, V_{j} \in L^{1}$ converges to the real potential $V \in L^{1}$ in $L^{1}$, then $\lim _{j \rightarrow \infty} \mathcal{N}_{f_{j}}\left(V_{j}\right)=\mathcal{N}_{f}(V)$ in $L^{1}$.

Proof. One has the estimate

$$
\begin{aligned}
& \left\|\mathcal{N}_{f_{j}}\left(V_{j}\right)-\mathcal{N}_{f}\left(V_{j}\right)\right\|_{L^{1}}= \\
& \quad \sup _{\|W\|_{L^{\infty}=1}}\left|\int\left(\mathcal{N}_{f_{j}}\left(V_{j}\right)-\mathcal{N}_{f}\left(V_{j}\right)\right) W d x\right|= \\
& \quad \sup _{\|W\|_{L^{\infty}=1}}\left|2 \operatorname{tr}\left(\left(f_{j}\left(H_{V_{j}}-\mu_{f_{j}}\left(V_{j}\right)\right)-f\left(H_{V_{j}}-\mu_{f}\left(V_{j}\right)\right)\right) W\right)\right| \leq \\
& 2 \mid f_{j}\left(H_{V_{j}}-\mu_{f_{j}}\left(V_{j}\right)\right)-f\left(H_{V_{j}}-\mu_{f}\left(V_{j}\right)\right) \|_{\mathcal{B}_{1}} \leq \\
& 2 \mid\left(f_{j}\left(H_{V_{j}}-\mu_{f_{j}}\left(V_{j}\right)\right)-f\left(H_{V_{j}}-\mu_{f_{j}}\left(V_{j}\right)\right)\right)\left(H_{V_{j}}-\rho\right)\left\|_{\mathcal{B}}\right\|\left(H_{V_{j}}-\rho\right)^{-1} \|_{\mathcal{B}_{1}},
\end{aligned}
$$

where $\rho<\rho_{\mathcal{C}}$, cf. Lemma 4.5. This leads to the estimate

$$
\begin{aligned}
& \left\|\mathcal{N}_{f_{j}}\left(V_{j}\right)-\mathcal{N}_{f}\left(V_{j}\right)\right\|_{L^{1}} \leq \\
& \quad 2 \mid\left(f_{j}\left(H_{V_{j}}-\mu_{f_{j}}\left(V_{j}\right)\right)-f\left(H_{V_{j}}-\mu_{f_{j}}\left(V_{j}\right)\right)\right)\left(H_{V_{j}}-\rho\right)\left\|_{\mathcal{B}}\right\|\left(H_{V_{j}}-\rho\right)^{-1 / 2} \|_{\mathcal{B}_{2}}^{2},
\end{aligned}
$$

Using (21), one estimates the second factor by

$$
\begin{aligned}
& \left\|\left(H_{V_{j}}-\rho\right)^{-1 / 2}\right\|_{\mathcal{B}_{2}} \leq \\
& \quad\left\|\left(H_{V_{j}}-\rho\right)^{-1 / 2}\left(H_{0}-\rho\right)^{1 / 2}\right\|_{\mathcal{B}}\left\|\left(H_{0}-\rho\right)^{-1 / 2}\right\|_{\mathcal{B}_{2}} \leq \sqrt{2}\left\|\left(H_{0}-\rho\right)^{-1 / 2}\right\|_{\mathcal{B}_{2}} .
\end{aligned}
$$

To estimate the first one, we write

$$
\begin{gathered}
\left\|\left(f_{j}\left(H_{V_{j}}-\mu_{f_{j}}\left(V_{j}\right)\right)-f\left(H_{V_{j}}-\mu_{f}\left(V_{j}\right)\right)\right)\left(H_{V_{j}}-\rho\right)\right\|_{\mathcal{B}} \leq \\
\sup _{t \in\left[\inf _{j} \operatorname{spec}\left(H_{V_{j}}\right), \infty[\right.} \mid\left(f_{j}\left(t-\mathcal{E}_{f_{j}}\left(V_{j}\right)\right)-f\left(t-\mathcal{E}_{f}\left(V_{j}\right)\right) \mid(t-\rho) \leq\right. \\
\sup _{t \in\left[\inf _{j} \operatorname{spec}\left(H_{V_{j}}\right), \infty[\right.} \mid\left(f_{j}\left(t-\mu_{f_{j}}\left(V_{j}\right)\right)-f\left(t-\mu_{f_{j}}\left(V_{j}\right)\right) \mid(t-\rho)+\right. \\
\sup _{t \in\left[\inf _{j} \operatorname{spec}\left(H_{V_{j}}\right), \infty[\right.} \mid\left(f\left(t-\mu_{f_{j}}\left(V_{j}\right)\right)-f\left(t-\mu_{f}\left(V_{j}\right)\right) \mid(t-\rho) .\right.
\end{gathered}
$$


The term (35) converges to zero due to (34) and $\mathcal{C} \subseteq \mathcal{D}_{2}$.

We consider the term (36). Lemma 4.7 yields that $\left\{\mu_{f_{j}}\left(V_{j}\right)\right\}_{j=1}^{\infty}$ and $\left\{\mu_{f}\left(V_{j}\right)\right\}_{j=1}^{\infty}$ converge to $\mu_{f}(V)$. Thus, the restriction of $f\left(\cdot-\mu_{f_{j}}\left(V_{j}\right)\right)-f(\cdot-$ $\left.\mu_{f}\left(V_{j}\right)\right)$ to finite intervals uniformly converges to zero by the continuity of $f$. On the other hand, $f$ decays at $\infty$ as $\frac{1}{t^{2}}$, thus, for large arguments $t$ the absolute value of $f\left(t-\mu_{f_{j}}\left(V_{j}\right)\right)-f\left(t-\mu_{f}\left(V_{j}\right)\right)$ becomes arbitrarily small uniformly in $j$. This altogether shows that (36) goes to zero.

Corollary 6.2. Let Assumptions [3.1, 3.3, 3.5, 3.8 and 3.14 be satisfied. Further, let $\mathcal{C}:=\left\{f_{j}\right\}_{j=1}^{\infty} \subset \mathcal{D}_{2}$, cf. Theorem 4.9, and $f \in \mathcal{D}$ such that condition (34) holds for every $a \in]-\infty,-1\left[\right.$. If $\left\{u_{j}\right\}_{j=1}^{\infty}, u_{j} \in L_{N}^{1}$ converges to the real function $u \in L_{N}^{1}$ in $L^{1}$, then $\lim _{j \rightarrow \infty} \Phi_{f_{j}}\left(u_{j}\right)=\Phi_{f}(u)$ in $L^{1}$.

Proof. By definition (31) we have

$$
\Phi_{f_{j}}\left(u_{j}\right):=\mathcal{N}_{f_{j}}\left(\Delta E+V_{x c}\left(u_{j}\right)-q \varphi\left(u_{j}\right)\right), \quad u_{j} \in L_{N}^{1}, \quad j=1,2, \ldots,
$$

where

$$
\varphi\left(u_{j}\right)=\widetilde{\varphi}+\mathcal{P}^{-1}\left(D-q u_{j}\right) .
$$

Since $\lim _{j \rightarrow \infty} \varphi\left(u_{j}\right)=\varphi(u)=\widetilde{\varphi}+\mathcal{P}^{-1}(D-q u)$ in $W^{1,2}$ one gets that $\lim _{j \rightarrow \infty} \varphi\left(u_{j}\right)=$ $\varphi(u)$ in $L^{1}$. Therefore $\lim _{j \rightarrow \infty} V_{j}=V$ in $L^{1}$, where

$$
V_{j}:=\Delta E+V_{x c}\left(u_{j}\right)-q \varphi\left(u_{j}\right) \quad \text { and } \quad V:=\Delta E+V_{x c}(u)-q \varphi(u) .
$$

Applying Lemma 6.1, we complete the proof.

Theorem 6.3. Let Assumptions [3.1, 3.3, 3.5, 3.8 and 3.14 be satisfied. Further, let $f \in \mathcal{D}$ and $\mathcal{C}:=\left\{f_{j}\right\}_{j=1}^{\infty} \subseteq \mathcal{D}_{2}$, cf. Theorem 4.9 such that $\mathcal{C}:=\left\{f_{j}\right\}_{j=1}^{\infty}$ obeys (34) for every $\left.a \in\right]-\infty,-1\left[\right.$. If $\left\{\left\{\varphi_{j}, u_{j}\right\}\right\}_{j=1}^{\infty}$ are solutions of the Kohn-Sham system with respect to the distribution function $f_{j}$, then there is a subsequence $\left\{\left\{\varphi_{k}, u_{k}\right\}\right\}_{k=1}^{\infty}$ which converges in $L^{\infty} \times L^{1}$ to a solution $\{\varphi, u\}$ of the Kohn-Sham system with distribution function $f$.

Proof. By Theorem 4.12 $\left\{\varphi_{j}, u_{j}\right\} \in W^{1,2} \times L^{1}$ is a solution of the Kohn-Sham system with respect to the distribution function $f_{j}$ if and only if $u_{j}=\Phi_{f_{j}}\left(u_{j}\right)$, $j=1,2, \ldots$ and the corresponding potential is given by $\varphi_{j}=\varphi\left(u_{j}\right)=\widetilde{\varphi}+$ $\mathcal{P}^{-1}\left(D-q u_{j}\right)$. According to Theorem 4.9 there are subsequences $\left\{u_{k}\right\}_{k=1}^{\infty}$ and $\left\{\varphi_{k}\right\}_{k=1}^{\infty}$ such that the following properties are satisfied:

- The sequence $\left\{u_{k}\right\}_{k}^{\infty}$ is bounded in $W^{1,2}$, obeys $u_{k} \in L_{N}^{1}$ and converges in $L^{1}$ to an element $u \in L_{N}^{1}$. 
- The sequence of potentials $\left\{\varphi_{k}\right\}_{k=1}^{\infty}$ converges in $L^{1}$ and, additionally, weakly in $W^{1,2}$ to an element $\varphi$.

By Theorem 4.12 the pair $\left\{\varphi_{k}, u_{k}\right\}$ is a solution of the Kohn-Sham system with distribution function $f_{k}$ if and only if $u_{k}$ is a fixed point of the map $\Phi_{k}$, i.e.

$$
u_{k}=\Phi_{f_{k}}\left(u_{k}\right), \quad k=1,2, \ldots .
$$

and the potential $\varphi_{k}$ is given by

$$
\varphi_{k}=\widetilde{\varphi}+\mathcal{P}^{-1}\left(D-q u_{k}\right)
$$

By $\lim _{k \rightarrow \infty} u_{k}=u$ in $L^{1}$ and Corollary 6.2 we get $u=\Phi_{f}(u)$ for $u \in L^{1}$. This shows that $u$ is a fixed point of $\Phi_{f}$. Moreover, one has $\varphi=\widetilde{\varphi}+\lim _{k \rightarrow \infty} \mathcal{P}^{-1}(D-$ $\left.q u_{k}\right)$ in $L^{\infty}$ which shows that $\varphi \in W^{1,2}$. By Theorem 4.12, the pair $\{\varphi, u\}$ is a solution of the Kohn-Sham system with distribution function $f$.

If the Kohn-Sham system with distribution function $f$ has several solutions, then it remains unclear to which of them a sequence of solutions of Kohn-Sham systems with distributions functions $f_{j}$ converges. However, if the exchange correlation term is absent, then the result can be improved.

Corollary 6.4. Let the assumptions of Theorem 6.3 be satisfied. If the exchange correlation term $V_{x c}$ is absent and if $\left\{\left\{\varphi_{j}, u_{j}\right\}\right\}_{j=1}^{\infty}$ are unique solutions of Kohn-Sham systems with distribution function $f_{j}$, then $\left\{\left\{\varphi_{j}, u_{j}\right\}\right\}_{k=1}^{\infty}$ converges in $L^{\infty} \times L^{1}$ to the unique solution $\{\varphi, u\}$ of the Kohn-Sham system with distribution function $f$.

Proof. Assume that the sequence $\left\{\left\{\varphi_{j}, u_{j}\right\}\right\}_{j=1}^{\infty}$ does not converge to $\{\varphi, u\}$. In this case there is a subsequence $\left\{\left\{\varphi_{k}, u_{k}\right\}\right\}_{k=1}^{\infty}$ converging in $L^{\infty} \times L^{1}$ to an element $\{\tilde{\varphi}, \tilde{u}\} \in L^{\infty} \times L^{1}$ which is different from $\{\varphi, u\}$. However, by Theorem 6.3 the pair $\{\tilde{\varphi}, \tilde{u}\}$ is a solution of the Kohn-Sham system with distribution function $f$. Since this Kohn-Sham system admits only one solution the solution $\{\tilde{\varphi}, \tilde{u}\}$ coincides with $\{\varphi, u\}$ - what is a contradiction.

Lemma 6.5. The function $f_{\beta}(x)=\frac{1}{\beta} \ln \left(1+e^{-\beta x}\right), x \in \mathbb{R}$, strictly decreases in $\beta \in] 0, \infty[$.

Proof. One calculates

$$
\frac{\partial}{\partial \beta} f_{\beta}(x)=-\frac{1}{\beta^{2}} \ln \left(1+e^{-\beta x}\right)-\frac{x}{\beta} \frac{e^{-\beta x}}{1+e^{-\beta x}},
$$


what immediately shows the assertion for $x \geq 0$. Putting $-\beta x=: \gamma$, the assertion for negative $x$ is equivalent to

$$
\frac{\gamma e^{\gamma}}{1+e^{\gamma}}<\ln \left(1+e^{\gamma}\right), \quad \gamma>0
$$

which follows from $\frac{\gamma e^{\gamma}}{1+e^{\gamma}}<\gamma=\ln \left(e^{\gamma}\right)<\ln \left(1+e^{\gamma}\right)$.

In order to apply this to the Kohn-Sham system at zero temperature, we show in the following that the corresponding distribution function satisfies the condition (34).

Lemma 6.6. Let $\left\{T_{j}\right\}_{j=1}^{\infty}$ be any positive sequence converging to zero. We set $f_{j}(x):=\frac{1}{\beta_{j}} \ln \left(1+e^{-\beta_{j} x}\right)$ where $\beta_{j}=\frac{1}{k T_{j}}$. Further, we set

$$
f(x):=\left\{\begin{array}{rll}
-x & : & x \leq 0 \\
0 & : & x>0
\end{array}\right.
$$

Then condition (34) is satisfied.

Proof. We have

$$
\begin{aligned}
\lim _{j \mapsto \infty} & \sup _{x \in[a, \infty[}\left|f_{j}(x)-f(x)\right| \theta(x) \leq \\
& \lim _{j \mapsto \infty} \sup _{a \leq x \leq 1}\left|f_{j}(x)-f(x)\right|+\lim _{j \mapsto \infty} \sup _{x \geq 1}\left|f_{j}(x)-f(x)\right| x
\end{aligned}
$$

for $a \leq-1$. Obviously we have

$$
\frac{1}{\beta} \ln \left(1+e^{-\beta x}\right) x \leq \frac{e^{-\beta x} x}{\beta}, \quad x \geq 1 .
$$

This shows that the second term of (38) tends to zero as $j \rightarrow \infty$. Further, it is almost obvious that $f_{j}(x)$ converges pointwise to the continuous function $f$ for $j \mapsto \infty$. Because the family $\left\{f_{\beta}\right\}_{\beta}$ is monotonously decreasing in $\beta$ by the preceding lemma, the convergence is uniform on bounded intervals by Dini's theorem. This proves that the first term of (38) tends to zero as $j \rightarrow \infty$.

\section{Acknowledgment}

H.C., H.N. and J.R. acknowledge support from the Danish F.N.U. grant Mathematical Physics and Partial Differential Equations. This work was initiated during a visit of K.H, H.N. and J.R. at the Department of Mathematical Sciences of the Aalborg University and they are thankful for kind hospitality extended to them during the work on this paper. 


\section{References}

[1] Olivier Bokanowski, José L. López, Óscar Sánchez, and Juan Soler. Long time behaviour to the Schrödinger-Poisson- $X^{\alpha}$ systems. In Mathematical physics of quantum mechanics, volume 690 of Lecture Notes in Phys., pages 217-232. Springer, Berlin, 2006.

[2] Olivier Bokanowski, José L. López, and Juan Soler. On an exchange interaction model for quantum transport: the Schrödinger-Poisson-Slater system. Math. Models Methods Appl. Sci., 13(10):1397-1412, 2003.

[3] M Cahay, M. McLennan, S. Datta, and M. S. Lundstrom. Importance of space-charge effects in resonant tunneling devices. Appl. Phys. Lett., 50(10):612-614, 1987.

[4] P. A. M. Dirac. Note on exchange phenomena in the Thomas atom. Proceedings Cambridge, 26:376-385, 1930.

[5] W. R. Frensley. Boundary conditions for open quantum systems driven far from equilibrium. Rev. Modern Phys., 62:745-791, 1990.

[6] H. Gajewski. Analysis und Numerik von Ladungstransport in Halbleitern. Report 6, WIAS, Berlin, 1993.

[7] P. Hohenberg and W. Kohn. Inhomogeneous electron gas. Phys. Rev., 136(3B):B864-B871, 1964.

[8] H.-Chr. Kaiser, H. Neidhardt, and J. Rehberg. Monotonicity properties of the quantum mechanical particle density. Preprint 1275, WIAS, Berlin, 2007.

[9] H.-Chr. Kaiser and J. Rehberg. About a one-dimensional stationary Schrödinger-Poisson system with Kohn-Sham potential. Z. Angew. Math. Phys., 50(3):423-458, 1999.

[10] H.-Chr. Kaiser and J. Rehberg. About a stationary SchrödingerPoisson system with Kohn-Sham potential in a bounded two- or three-dimensional domain. Nonlinear Anal., 41(1-2, Ser. A: Theory Methods):33-72, 2000.

[11] T. Kato. Perturbation theory for linear operators. Die Grundlehren der mathematischen Wissenschaften, Band 132. Springer-Verlag New York, Inc., New York, 1966. 
[12] T. Kerkhoven. Mathematical modeling of quantum wires in periodic heterojunction structures. In Semiconductors Part II, volume 59 of The IMA Volumes in Mathematics and its Applications, pages 237-253. Springer, New York, 1994.

[13] T. Kerkhoven. Numerical nanostructure modeling. Z. Angew. Math. Mech., 76(Suppl. 2):297-300, 1996.

[14] W. Kohn and L. J. Sham. Self-consistent equations including exchange and correlation effects. Phys. Rev., 140(4A):A1133-A1138, 1965.

[15] Elliott H. Lieb and Barry Simon. The Hartree-Fock theory for Coulomb systems. Comm. Math. Phys., 53(3):185-194, 1977.

[16] P.-L. Lions. Solutions of Hartree-Fock equations for Coulomb systems. Comm. Math. Phys., 109(1):33-97, 1987.

[17] N. D. Mermin. Thermal properties of the inhomogeneous electron gas. Phys. Rev., 137(5A):A1441-A1443, 1965.

[18] F. Nier. Etude mathématique et numêrique de modèles cinétiques quantiques issus de la physique des semi-conducteurs. Master's thesis, Ecole Polytechnique, 1991.

[19] F. Nier. A variational formulation of Schrödinger-Poisson systems in dimension $d \leq 3$. Comm. Partial Differential Equations, 18(7-8):11251147, 1993.

[20] E. Prodan and P. Nordlander. On the Kohn-Sham equations with periodic background potentials. J. Statist. Phys., 111(3-4):967-992, 2003.

[21] P. N. Racec, U. Wulf, and J. Kučera. Integration of quantum transport models in classical device simulators. Solid-State Electronics, 44(5):881$886,2000$.

[22] Óscar Sánchez and Juan Soler. Long-time dynamics of the SchrödingerPoisson-Slater system. J. Statist. Phys., 114(1-2):179-204, 2004.

[23] J. C. Slater. A Simplification of the Hartree-Fock Method. Phys. Rev., 81(3):385-390, 1951.

[24] E. Zeidler. Nonlinear functional analysis and its applications. II/B. Springer-Verlag, New York, 1990. Nonlinear monotone operators, Translated from the German by the author and Leo F. Boron. 
[25] B. Zimmermann, E. E. Marclay, M. Ilegems, and P. Guéret. Self-consistent calcultions of tunneling currents in $n^{+}-\mathrm{GaAs} / i$ $\mathrm{Al}_{x} \mathrm{Ga}_{1-x} \mathrm{As} / n^{+}$-GaAs structures and comparison with measurements. J. Appl. Phys., 64(7):3581-3588, 1988. 